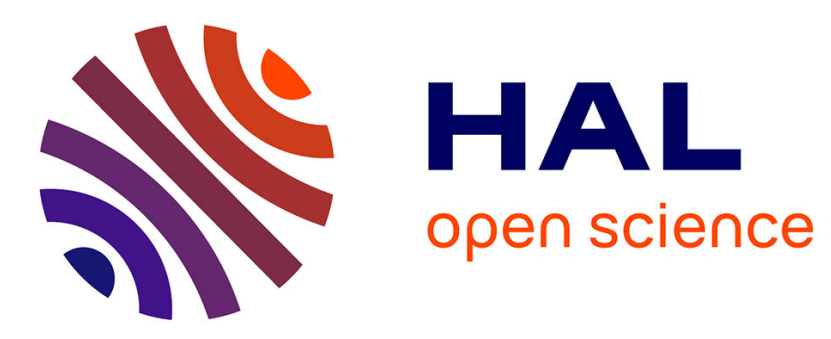

\title{
Photon localization in disorder-induced periodic multilayers
}

B. van Tiggelen, Adriaan Tip

\section{To cite this version:}

B. van Tiggelen, Adriaan Tip. Photon localization in disorder-induced periodic multilayers. Journal de Physique I, 1991, 1 (8), pp.1145-1154. 10.1051/jp1:1991196 . jpa-00246400

\section{HAL Id: jpa-00246400 https://hal.science/jpa-00246400}

Submitted on 1 Jan 1991

HAL is a multi-disciplinary open access archive for the deposit and dissemination of scientific research documents, whether they are published or not. The documents may come from teaching and research institutions in France or abroad, or from public or private research centers.
L'archive ouverte pluridisciplinaire HAL, est destinée au dépôt et à la diffusion de documents scientifiques de niveau recherche, publiés ou non, émanant des établissements d'enseignement et de recherche français ou étrangers, des laboratoires publics ou privés. 
Classification

Physics Abstracts

$05.60-63.20 \mathrm{P}$

\title{
Photon localization in disorder-induced periodic multilayers
}

\author{
Bart A. van Tiggelen and Adriaan Tip \\ FOM-Institute for Atomic and Molecular Physics, Kruislaan 407, 1098 SJ Amsterdam, The \\ Netherlands
}

(Received 28 February 1991, accepted 26 April 1991)

\begin{abstract}
Résumé. - Nous avons examiné l'influence du désordre sur un système stratifié et diélectrique, à une dimension. Le système est d'origine périodique. Des simulations numériques ont été effectuées et devraient être confrontées à des prédictions théoriques. L'exposant de Lyapunov est non analytique aux bords du spectre. Nous avons constaté que les anomalies qui pourraient se trouver au centre des bandes sont beaucoup plus nettes que celles dans le modèle d'Anderson.

Abstract. - We studied the influence of randomness on a periodic dielectric 1D stratified system. Numerical simulations are performed, and are compared to theoretical predictions. Non-analytic behaviour is found at the band edges. The anomalies that can arise in the middle of a band turn out to be far more pronounced than the ones in the Anderson-model.
\end{abstract}

\section{Introduction.}

Localization is the phenomenon that a wave becomes spatially localized in the presence of disorder. Since its introduction by Anderson [1] in 1958 there has been much progress in its understanding. Criteria [2] and scaling theories [3] were developed, predicting the occurrence of localization in one and two dimensions regardless of the degree of disorder, and the presence of a mobility edge in three dimensions [4]. Later, Vollhardt and Wölfe [5], presented a microscopic localization theory, where the constructive interference of timereversed waves, known as «weak localization », was taken to be responsible for localization. In favour of their treatment was its agreement with scaling theory, and the fact that the existence of weak localization was reported in light reflection experiments [6]. On the other hand, techniques first developed by Furstenberg [7] have been used to treat localization in one dimension in a mathematical rigorous way $[8,9]$, without any reference to perturbation theory. This approach does not work in three dimensions, although some results have been obtained for this case [10]. The exact conditions for the occurrence of localization in one dimension are subtle, but, loosely speaking, the statement is that states are localized for " almost any » energy and " almost any » realization of the system, provided the probability measure, determining nature and strength of the disorder, is ergodic. This makes 1D localization very suitable for numerical simulation, for the ergodicity demand is justified in most cases [11]. 
The physical quantity that determines the exponential decay of the wave function, is the (upper) Lyapunov exponent. Given a set of random matrices $\left\{\mathbf{M}_{l}(\omega)\right\}$ it is defined as

$$
\gamma \equiv \lim _{\gamma \rightarrow \infty} \frac{1}{N} \log \left\|\mathbf{M}_{1}(\omega) \cdot \mathbf{M}_{2}(\omega) \cdot \mathbf{M}_{N}(\omega)\right\|
$$

and is, for almost any energy [12], a positive quantity almost surely independent of $\omega, \omega$ indicating a realization of the system. A very useful result is the Thouless formula [13], relating the Lyapunov exponent to the density of states (DOS) by means of a dispersion relation. It finds its origin in the fact that both quantities are real and imaginary part of one analytic function. This fundamental relation may be taken to be responsible for anomalous behaviour of the Lyapunov exponent on band edges of the parent system [14]. In more dimensions, a simple Thouless formula no longer holds, but, nevertheless, the DOS may be an appealing pathway to find criteria for localization $[3,15,16]$.

To describe the one dimensional localization of electrons, most workers treat the Anderson Model with diagonal disorder, but the concept applies to any problem involving random transfer matrices, and thus also for the transmission of light in random layers. Consequently, a sufficiently large stack of dielectric layers, with random thickness and/or index of refraction will act as a perfect mirror "almost surely ", that is always except for a countable number of energies and a set of realizations with probability zero, such as periodic stacks. Recently, the study of quasi-periodic stacks, within the context ob both electron $[17,18]$ and light transmission $[19,20,21]$, has become very popular. It has been realized that these deterministic structures can also give rise to localized states, and as such, form an important regime between order and disorder.

This paper deals with the influence of periodicity of the parent system on the Lyapunov exponent. The band structure of the parent system will have a drastic influence on this quantity. The system we describe is of the form $A B_{1} A B_{2} A B_{3} .$. where the indices of refraction of the $B_{l}$ 's will be given random distributions of different kind. The non-analytic behaviour at different band edges is investigated, and large dips are discovered near special energies, for which the wavelength matches with both $A$ and $\left\langle B_{l}\right\rangle$. In principle, this phenomenon can be used to construct sharp filters. In both cases, the numerical simulations show universal behaviour of the Lyapunov exponent. Excellent agreement is found with the theory developed by Kappus and Wegner [22], Lambert [23] and Derrida and Gardner [14].

\section{The model.}

We consider the propagation of electromagnetic waves in a stack of dielectric layers, and confine ourselves to the case of normal incidence of linearly polarized light. To this end we define the state vector $\mathbf{C} \equiv\left(\varepsilon^{1 / 2} E_{z}, B_{y}\right)$, with $E_{z}$ and $B_{1}$ the components of the electric and magnetic field along the $z$-axis and $y$-axis. The $x$-axis is located along the slab, in the direction of propagation; $\varepsilon$ is the dielectric constant. Maxwell's equations imply the following dynamics:

$$
\frac{\partial \mathbf{C}}{\partial t}=-i\left(\begin{array}{cc}
0 & \Gamma^{-1} p \\
p \Gamma^{-1} & 0
\end{array}\right) \cdot \mathbf{C} .
$$

Here is $p \equiv-i \partial / \partial x, \Gamma \equiv \varepsilon^{1 / 2}$ and our units are such that the speed of light in vacuum, $c_{0}=1$. If we insert modes $\exp (-i E t)$ the solution of equation (2) in the $n$-th layer is

$$
\mathbf{C}_{n}(x)=\alpha_{n}\left(\begin{array}{r}
1 \\
-1
\end{array}\right) \mathrm{e}^{-\imath k_{n}\left(x-x_{n}\right)}+\beta_{n}\left(\begin{array}{l}
1 \\
1
\end{array}\right) \mathrm{e}^{i k_{n}\left(x-x_{n}\right)}
$$


with $k_{n} \equiv E \Gamma_{n}$. The continuity of $E_{z}$ and $B_{y}$ on the interface of two layers fixes the transfer matrix:

$$
\begin{gathered}
\left(\begin{array}{l}
\alpha_{n+1} \\
\beta_{n+1}
\end{array}\right)=\mathbf{M}(n) \cdot\left(\begin{array}{l}
\alpha_{n} \\
\beta_{n}
\end{array}\right), \\
\mathbf{M}(n)_{11}=\mathbf{M}(n)_{22}^{*}=\frac{\Gamma_{n}+\Gamma_{n+1}}{2 \Gamma_{n}} \mathrm{e}^{-i k_{n+1}\left(x_{n+1}-x_{n}\right)}, \\
\mathbf{M}(n)_{12}=\mathbf{M}(n)_{21}^{*}=\frac{\Gamma_{n+1}-\Gamma_{n}}{2 \Gamma_{n}} \mathrm{e}^{-\imath k_{n+1}\left(x_{n+1}-x_{n}\right)}
\end{gathered}
$$

The layers with even $n$ are taken identical and fixed, and for simplicity referred to as vacuum. From now on the index $n$ counts the vacuum layers only. The wave function at « site " $n$ determines the electromagnetic field in the $n$-th vacuum layer; $\Gamma_{n}$ is the reciproke speed of light inbetween the $n$-th and $(n+1)$-th vacuum layer. The transfer matrix mapping one vacuum layer onto the next one is given by

$$
\mathbf{M}(n \rightarrow n+1)=\left(\begin{array}{rr}
a_{n} & i q_{n} \\
-i q_{n} & a_{n}^{*}
\end{array}\right),
$$

where $a_{n}$ and $q_{n}$ are given by:

$$
\begin{aligned}
& a_{n}=\mathrm{e}^{-i y}\left[\cos y \Gamma_{n}-i \frac{\Gamma_{n}^{2}+1}{2 \Gamma_{n}} \sin y \Gamma_{n}\right] ; \\
& q_{n}=\mathrm{e}^{-i y} \frac{1-\Gamma_{n}^{2}}{2 \Gamma_{n}} \sin y \Gamma_{n} .
\end{aligned}
$$

All layers are assumed to have common width $d ; y \equiv E d$ is a dimensionless energy parameter. The exponent in $q_{n}$ can be eliminated by a ( $n$-independent) unitary transformation. The matrix has determinant 1 . If all the non-vacuum layers have the same value for $\Gamma$ a superlattice structure [24] is present, and the "photon " band structure is determined by the criterium that both eigenvalues have unity norm, which, in turn, implies $|\operatorname{Re} a| \leqslant 1$. Although in the perfect periodic case it is possible to write down an "Anderson-type » of equation

$$
\left(\begin{array}{c}
\alpha_{n+1} \\
\beta_{n+1}
\end{array}\right)+\left(\begin{array}{c}
\alpha_{n-1} \\
\beta_{n-1}
\end{array}\right)=2 \operatorname{Re} a\left(\begin{array}{c}
\alpha_{n} \\
\beta_{n}
\end{array}\right),
$$

this formula no longer holds if the Bloch symmetry is broken, which is true if disorder is introduced, and $\Gamma_{n} \equiv \Gamma_{0}+\delta_{n}$. We do not introduce randomnes in the width of the layers [25]. The $\delta_{n}$ 's are assumed to be mutually independent, identically distributed stochastic variables with zero mean, and deviation $\sigma$. This guarantees the stochastic process to be ergodic [11]. Consequently, the Lyapunov exponent can be calculated numerically on the computer by generating only one realization $\left\{\Gamma_{n}\right\}$, and then evaluate the product in equation (1). Many authors made use of the stationarity of the underlying probability measure, to find the behaviour of both the Lyapunov exponent and the DOS, often in the limit of low disorder. We investigated the range of validity of these predictions, and find good agreement. Special interest is devoted to energies corresponding to the band edges of the parent system and special « Fabry-Perot » energies. 
2.1 THE BAND EDGES. - Many workers have predicted the anomalous behaviour of the Lyapunov exponent on a band edge [26-28]. A very elegant renormalization treatment was given by Bouchaud and Daoud [29], resulting in $\gamma \sim \sigma^{23}$ at the band edges. A straightforward application of their method to our random matrix yields. in the limit $\sigma, \Delta \rightarrow 0$

$$
\gamma(\Delta, \sigma)=\sigma^{23} F_{\mathrm{b}}\left(\frac{\Delta}{\sigma^{4 / 3}}\right)
$$

Here $\Delta \equiv y-y_{\text {edge }}$. On the band side of the edge we expect the anomalous behaviour to disappear and to go over into $\gamma \sim \sigma^{2}$. Hence $F_{\mathrm{b}}(\eta) \sim 1 / \eta$. On the gap side the Lyapunov exponent approaches the logarithm of the largest eigenvalue of the transfer matrix, which implies $F_{\mathrm{b}}(\eta) \sim \sqrt{\eta}$. However, this does not fix $F_{\mathrm{b}}(\eta)$ completely. Using the degenerate perturbation theory of Kappus and Wegner [22]. Derrida and Gardner [14] calculated the function $F_{\mathrm{b}}(\eta)$ for the Anderson model:

$$
F_{\mathrm{b}}(\eta)=\frac{1}{2} \frac{\int_{0}^{\infty} \mathrm{d} t t^{12} \mathrm{e}^{-t^{3} 6+2 \eta t}}{\int_{0}^{\infty} \mathrm{d} t t^{-1,2} \mathrm{e}^{-t^{3} 6+2 \eta t}}
$$

If we compare our model with the Anderson model, with energy $E$ and diagonal disorder $V_{n}$. identifying as in equation (4)

$$
E+V_{n}=2 \operatorname{Re} a\left(y, \Gamma_{n}\right)=2 \operatorname{Re} a\left(y, \Gamma_{0}\right)+2 \frac{\partial \operatorname{Re} a}{\partial \Gamma}\left(y, \Gamma_{0}\right) \delta_{n},
$$

then we can show (see the Appendix) that, within the scaling assumption of Derrida and Gardner, equation (6) is valid for our model as well. This is not trivial, for we have already indicated that a rigorous mapping onto the Anderson model breaks down in the presence of randomness. The figures 1 compare the scaling prediction to numerical simulations, performed on a VAX 3100 station. The distribution of the $\delta$ s was taken to be Gaussian, and every data point was generated independently from all the others. The agreement between the scaling result and the simulations is very good, over three decades in disorder, and we conclude that scaling theory provides an accurate description of the Lyapunov exponent near the edges of a band.

2.2 FABRY-PEROT RESONANCES. - In this section we let the average index of refraction $\Gamma_{0}$ of the random layers be rational. In that case the band structure of the parent system itself becomes periodic in energy. Special energies $E$ exist, to which we shall refer as Fabry-Perot energies, for which a multiple of the wavelength fits exactly in both the vacuum layer and the average of the random layer. Such an energy is guaranteed to be located in the band of the parent system since then $\operatorname{Re} a= \pm 1$. Using again the procedure of Bouchaud and Daoud [29] it is easily established that the scaling limit of small $\sigma$ and $\Delta$ takes the form:

$$
\gamma(\Delta, \sigma)=\sigma^{2} F_{\mathrm{d}}\left(\frac{\Delta}{\sigma^{2}}\right) .
$$

Now, $\Delta \equiv y-n \pi$. We have taken $\Gamma_{0}=m / n$. The function $F_{\mathrm{a}}(\eta)$ can be calculated using the degenerate perturbation theory developed by Kappus and Wegner [22]. Because the transfer matrix has determinant 1 we have a conservation law $\left|\alpha_{n}\right|^{2}-\left|\beta_{n}\right|^{2}=$ constant. For a sem1infinite system it is easily seen that this constant is zero, so that we can take $\alpha_{n} / \beta_{n}=i \mathrm{e}^{i \phi_{n}}$ 


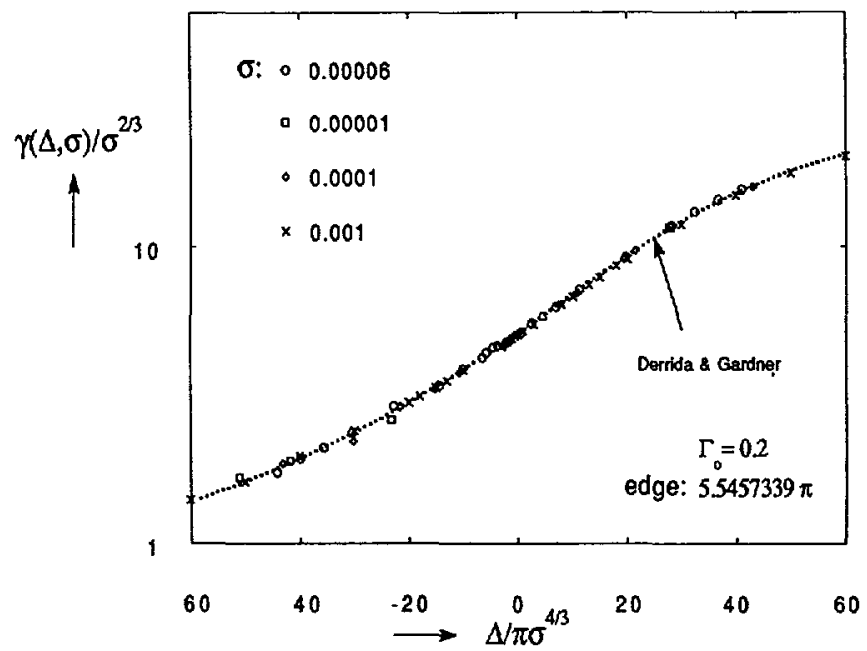

a)

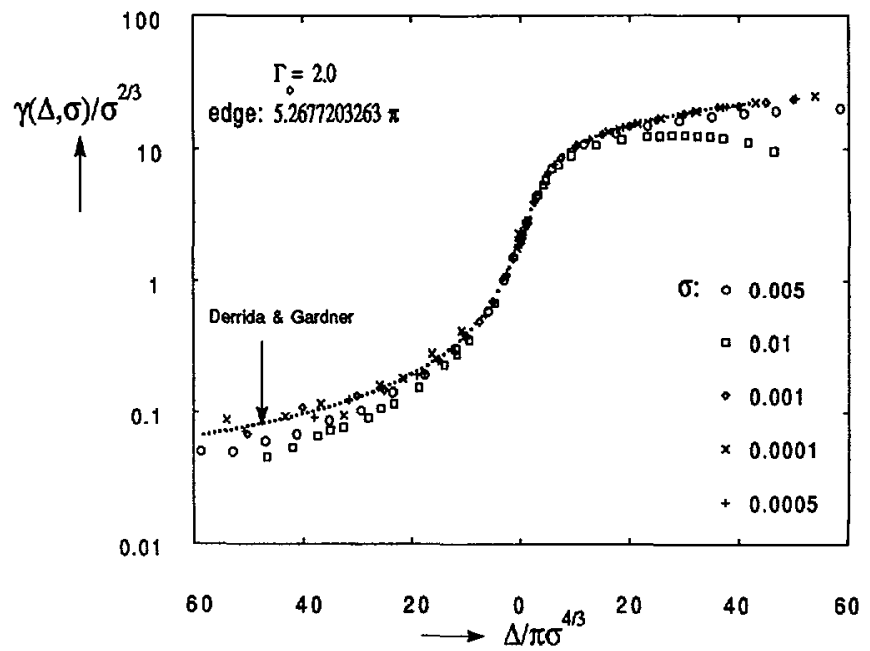

b)

Fig. 1. - Numerical simulation of the Lyapunov exponent near a band edge, using $5 \times 10^{5}$ layers. A Gaussian distribution with deviation $\sigma$ was used. The scaling behaviour of equation (5) is confirmed. The dotted line is the theory of reference [14].

Equation (3) results in

$$
\mathrm{e}^{i \phi_{n+1}}=\frac{\mathrm{e}^{t\left(\theta_{n}+\phi_{n}\right)}+S_{n}}{\mathrm{e}^{-i \theta_{n}}+S_{n} \mathrm{e}^{i \phi_{n}}},
$$

where we defined $S_{n} \equiv q_{n} /\left|a_{n}\right|$ and $\mathrm{e}^{\prime t_{n}} \equiv a_{n} /\left|a_{n}\right|$. With $\mu_{\jmath} \equiv\left\langle\mathrm{e}^{l \jmath \phi}\right\rangle$ and $a_{k l} \equiv\left\langle S^{h} \mathrm{e}^{i \ell \theta}\right\rangle$ it follows [23] that

$$
\begin{gathered}
\sum_{j=1}^{\infty} W_{\ell j} \mu_{j}=a_{\ell \ell}, \\
W_{\ell j}=\delta_{\ell j}-\sum_{k \geqslant \ell-j}^{\ell}(-1)^{j+k-\ell} \frac{\ell(\ell+k-1) !}{(\ell-k) ! k !(j+k-\ell) !} a_{2 k+j-\ell, \ell+j}
\end{gathered}
$$


(the paper of Lambert [23] contains an erroneous factor $\ell$ ! instead of $\ell$ in the nominator of Eq. (10)). We search for a scaling solution of the form of equation (8). To this end we write $y=n \pi+\lambda^{2} s$ and $\Gamma=m / n+\lambda t$, with $\lambda$ small. The variables $a$ and $q$ in equation (3) can be expanded as follows

$$
a=(-1)^{m+n}\left[1-i A \lambda-i B \lambda^{2}-C \lambda^{2}\right], \quad q=(-1)^{m}\left[D \lambda+E \lambda^{2}\right],
$$

with $A=\pi\left(m^{2}+n^{2}\right) t / 2 m, B=\left(m^{2}+3 n^{2}\right) s / 2 n^{2}+\pi\left(m^{2}-n^{2}\right) t^{2} n / 2 m^{2}, C=n^{2} \pi^{2} t^{2 / 2}$, $D=\pi\left(m^{2}-n^{2}\right) t / 2 m$ and $E=\left(m^{2}-n^{2}\right) s / 2 m^{2}+\pi\left(m^{2}+n^{2}\right) t^{2} n^{2} 2 m^{2}$. We find to order $\lambda^{2}$

$$
\begin{gathered}
a_{0 k}=(-1)^{k(m+n)}\left[1-\left(\frac{1}{2} k^{2}\left\langle A^{2}\right\rangle+i k\langle B\rangle\right) \lambda^{2}\right] \\
a_{1 k}=(-1)^{m+k(n+m)}(\langle E\rangle-\imath k\langle A D\rangle) \lambda^{2}, \quad a_{2 h}=(-1)^{k(n+m)}\left\langle D^{2}\right\rangle \lambda^{2}
\end{gathered}
$$

All other $a_{\ell_{k}}$ are higher order in $\lambda$ and are ignored. The matrix elements $W_{\ell_{1}}$, again to order $\lambda^{2}$, can now be obtained from

$$
W_{\ell, \ell}=1-a_{0,2 \ell}+\ell^{2} a_{2,2 \ell}, \quad W_{\ell, \ell \pm 1}= \pm \ell a_{1,2 \ell \pm 1}, \quad W_{\ell, \ell \pm 2}=-\frac{1}{2} \ell(\ell \pm 1) a_{2} 2 \ell \pm 2 .
$$

These elements are seen to be proportional to $\lambda^{2}$ so that we write $W_{\ell_{j}} \equiv Q_{\ell_{j}} \lambda^{2}$; all other elements are zero to order $\lambda^{2}$. Furthermore $a_{11} \equiv v_{1} \lambda^{2}, a_{22} \equiv v_{2} \lambda^{2}$, and $v_{1}=0$ for $t \geqslant 3$. The moments $\left(\mu_{1}, \mu_{2}, \ldots\right) \equiv \mu$ are seen to follow from the equation: $\mathbf{Q} \cdot \mu=\mathbf{v}$ Since $1 \geqslant|\mu,| \rightarrow 0$ as $j \rightarrow \infty$ it can be solved accurately, choosing a sufficiently large dimensional1ty $M$ for the matrix $Q$. We find, with $\eta=s / t^{2}$

$$
F_{\mathrm{d}}(\eta)=\frac{1}{2 \sigma^{2}}\left\langle\log \left|a+q \mathrm{e}^{-\imath \phi}\right|^{2}\right\rangle=\frac{D^{2}}{2 t^{2}}+\operatorname{Re}\left[\frac{E}{t^{2}} \mu_{1}-\frac{A D}{t^{2}} l \mu_{1}-\frac{D^{2}}{2 t^{2}} \mu_{2}\right]+\mathcal{O}(\lambda) .
$$

The figures 2 display the result of simulations, for different disorders, as well as a calculation based on equations (12) and (13). The remarkable dip in the Lyapunov exponent is known as an anomaly, and turns out to be slightly redshifted with respect to the Fabry-Perot energy $n \pi$. From the simulations we infer that the dip disappears for large disorders and the scaling result becomes inaccurate. Because the theory still involves the diagonalization of a matrix of infinite dimensionality, it is not clear to us whether the scaling result exactly vanishes in the center of the anomaly. Clearly, we expect a very large stack, generated according to a similar procedure, to act as a narrow bandpass filter. This anomaly thus may have an important application [30].

The theory demonstrates the irrelevance of the nature of the probability distribution. provided the scaling hypothesis is satisfied, that is, the deviation $\sigma$ must exist and be sufficiently small. This is also true for a distribution of the kind $\Gamma=m / n \pm \sigma$, both with probability $1 / 2$. Numerical results for a stack, generated according to such a distribution, are presented in figure 3. They are found to coincide nicely with the scaling solution.

\section{Binary distribution.}

The one dimensional Anderson model with diagonal binary disorder was studied extensively by Nieuwenhuizen [31] and others. A singular power-law behaviour of the density of states was predicted, as well as the existence of extremely narrow and pronounced dips in the Lyapunov exponent. These dips were referred to as «islands » in order to distinguish them 


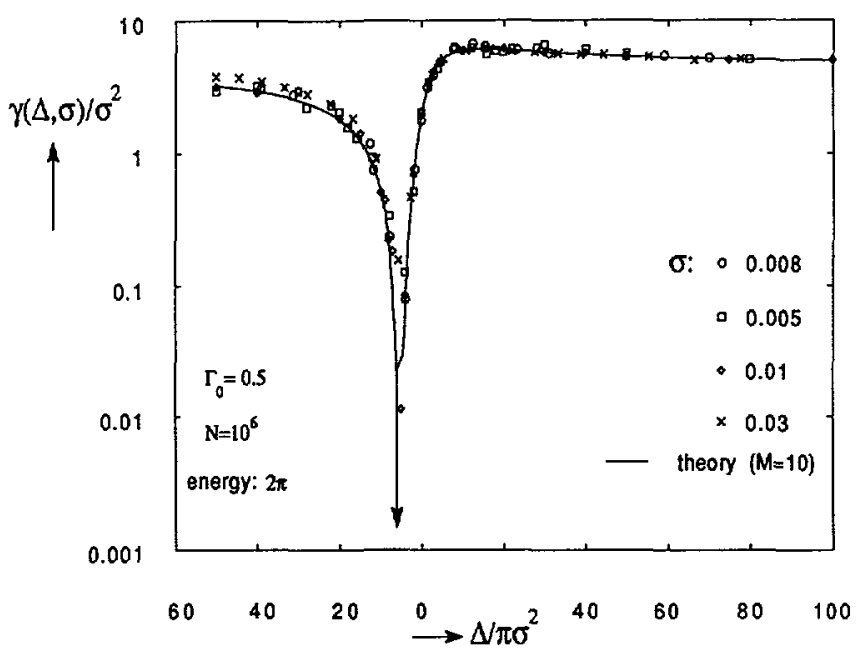

a)

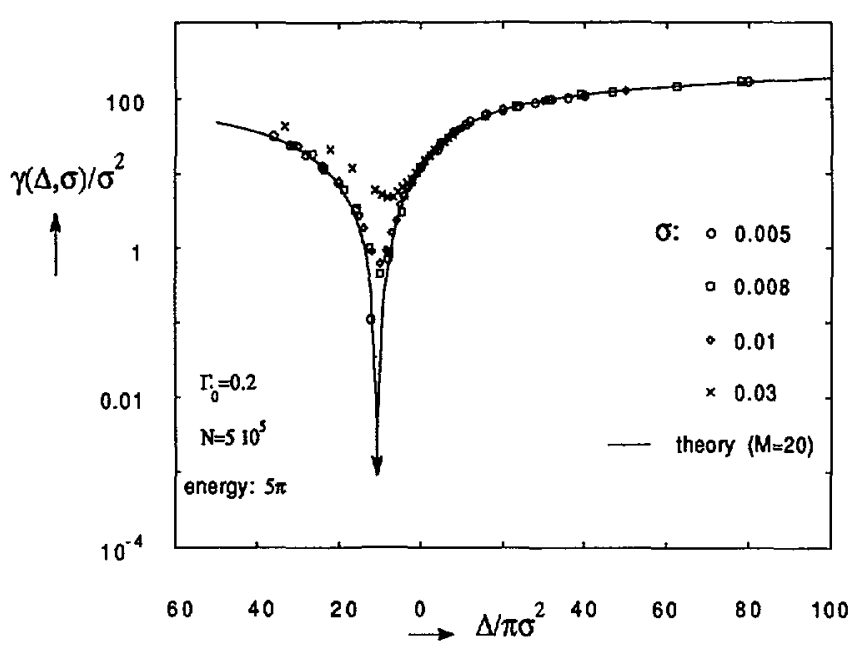

b)

Fig. 2. - Numerical simulation of the Lyapunov exponent near 2 special " Fabry-Perot energies » using a Gaussian distribution for the index of refraction. The line is the application of equations (10)-(13). The dimension of the matrix $\mathbf{Q}$ is indicated by $M$, the number of layers by $N$. Every data-point represents an independent simulation.

from anomalies which are not specific for binary distributions. Very recently, Crisanti [32] investigated a binary optical multilayer and found zero Lyapunov exponents on special FabryPerot energies, for any disorder. We demonstrate the applicability of the theory of section 2.2 for any dielectric binary multilayer. We consider again the sequence $A B_{1} A B_{2} \ldots$ and assume that $B_{i}=A(\Gamma=1)$ with probability $1-p$ and $B_{i}=B_{0}(\Gamma=\Gamma(B))$ with probability $p$. Furthermore $a$ and $q$ are the parameters defined in equation (3) corresponding to $\Gamma(B)$. With the notation of section 2.2 it is easily shown that

$$
a_{k l}=\delta_{0 k}(1-p) \mathrm{e}^{-2 l_{i y}}+p\left(\frac{q}{|a|}\right)^{k}\left(\frac{a}{|a|}\right)^{\ell}
$$




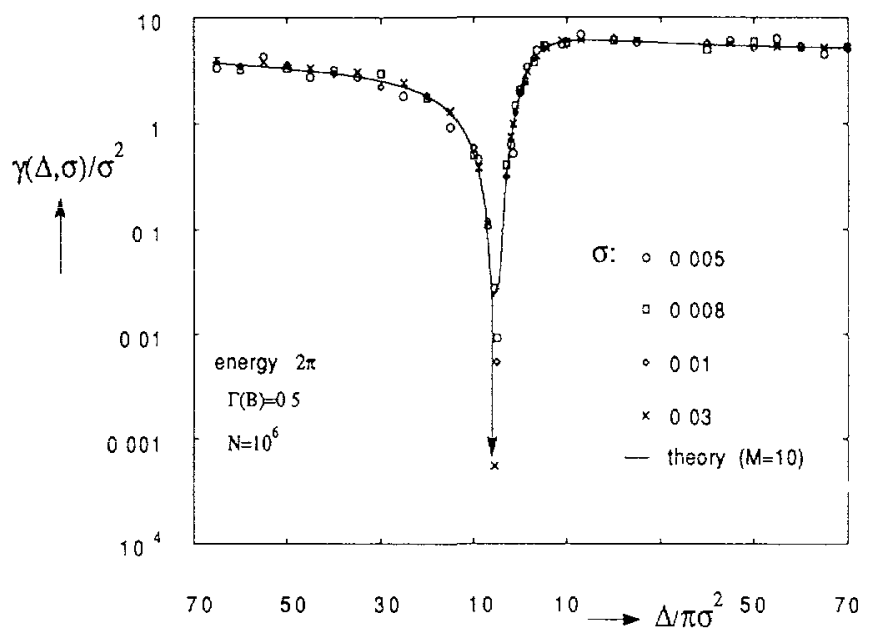

Fig. 3 - As in figure 2, but now the $\Gamma_{n}$ are distributed according to $05 \pm \sigma$ both with probabilit 12

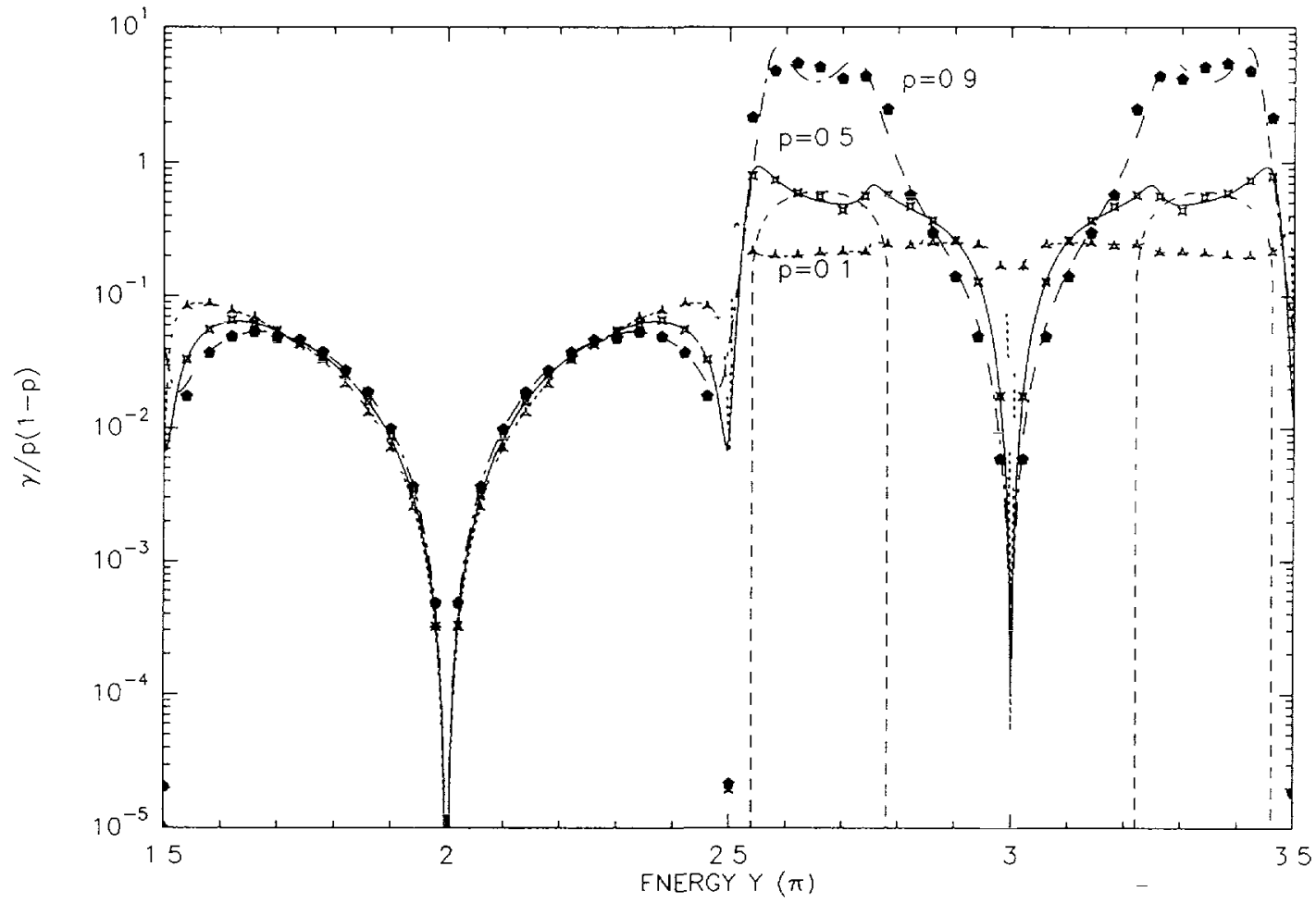

F1g. 4. - The Lyapunov exponent for the optical multilayer with binary distribution. The lines represent evaluation of equations (15) and (10). The matrix $W$ has been given dimension $M=2$. the data-points are simulations on $5 \times 10^{5}$ layers The dashed line is the exponent for the periodic stack $A B_{0} A B_{n} . \quad(p=1)$. 
The various $\mu_{t}$ can be found using equation (10). The Lyapunov exponent is then calculated as follows

$$
\gamma=\left\langle\log \left|\frac{\alpha_{n+1}}{\alpha_{n}}\right|\right\rangle=\operatorname{Re}\left\langle\log \left(a+q \mathrm{e}^{-i \phi}\right)\right\rangle=p \log |a|-\sum_{j=1}^{\infty} \frac{(-1)^{j}}{j} \operatorname{Re}\left[a_{j j} \mu_{j}\right] .
$$

Figure 4 shows a comparison of a simple version of the theory $(M=2)$ with a numerical simulation on $5 \times 10^{5}$ layers. On the vertical axis we plotted $\gamma(p) / p(1-p)$, knowing that the denominator is proportional to the variance of the binary distribution. Perfect agreement with theory was established for $M=10$, but the outcome for $M=2$ is still surprisingly satisfactory. In addition we plotted the Lyapunov exponent for the periodic system $B_{0} A B_{0} A B_{0} \ldots$, corresponding to $p=1$. For energies in the spectrum of this system, we infer $\gamma \sim \sigma^{2}$ and anomalous behaviour seems absent. We have not found evidence for the presence of islands, but this may well be caused by insufficient energy resolution. Because the binary distribution is attractive from an experimental point of view, random multilayers, generated accordingly, may find an important application in X-ray optics [33].

\section{Acknowledgments.}

We acknowledge Ad Lagendijk, Theo Nieuwenhuizen, J. Luck and Jan Verhoeven for useful discussions. This work is part of the research program of the Stichting voor Fundamenteel Onderzoek der Materie (FOM) and was made possible by financial support from the Nederlandse Organisatie voor Wetenschappelijk Onderzoek (NWO).

\section{Appendix.}

We give an outline of the proof that the analytical scaling result for the Lyapunov exponent, equation (6), applied to the one dimensional Anderson model, also holds near the band edges of the dielectric multilayer. To this end we show that the random matrices

$$
\mathbf{M}_{1}=\left(\begin{array}{cc}
a_{n} & i q_{n} \\
-i q_{n} & a_{n}^{*}
\end{array}\right) \text { and } \mathbf{M}_{2}=\left(\begin{array}{cc}
2 \operatorname{Re} a_{n} & -1 \\
1 & 0
\end{array}\right)
$$

have the same Lyapunov exponent near the band edge, in the scaling limit of Derrida and Gardner [14]. $\mathbf{M}_{2}$ is in "Anderson » form if the identification equation (7) is made. The change of base $\mathbf{U}_{n}$, mapping $\mathbf{M}_{1}$ onto $\mathbf{M}_{2}$

$$
\mathbf{U}_{n} \cdot \mathbf{M}_{2} \cdot \mathbf{U}_{n}^{-1}=\mathbf{M}_{1}
$$

is given by

where

$$
\mathbf{U}_{n}=\left(\begin{array}{cc}
s_{n} & s_{n} z_{n} \\
-1 / 2 s_{n} z_{n} & 1 / 2 s_{n}
\end{array}\right)
$$

$$
s_{n}=\sqrt{2 i q_{n} /\left(a_{n} z_{n}+1\right)}, \quad z_{n}=i \operatorname{Im} a_{n}+\sqrt{1-\left(\operatorname{Im} a_{n}\right)^{2}} .
$$

If we denote the wave function corresponding to the transfer matrix $M_{2}$ by the vector $\left(\tilde{\alpha}_{n}, \tilde{\beta}_{n}\right)$ and $R_{n} \equiv \tilde{\alpha}_{n} / \tilde{\beta}_{n}$, we find, after some algebra

$$
\frac{\alpha_{n+1}}{\alpha_{n}}=a_{n}+\left(a_{n}+z_{n}^{-1}\right) \frac{R_{n}-z_{n}}{R_{n}+z_{n}}
$$


Notice that $\left\langle\log \left|\alpha_{n+1} / \alpha_{n}\right|\right\rangle$ equals the Lyapunov exponent of $\mathbf{M}_{1}$, whereas $\left\langle\log \left|R_{n}\right|\right\rangle$ is the one for $\mathbf{M}_{2}$. Next, a scaling expansion has to be employed for $a_{n}, z_{n}$ and $R_{n}$. Following reference [14] we must use $1-y_{\text {edge }} \sim \lambda^{43}, \Gamma_{n}-\Gamma_{n} \sim \lambda$, and $R_{n}= \pm 1+\lambda^{23} r$, with $\lambda$ small. To lowest order in $\lambda$ it is easily inferred that only the scaling of $R_{n}$ matters in equation (A1). Consequently $\log \left|\alpha_{n+1} / \alpha_{n}\right|=\lambda^{23} K r$. Fl, 11 equation (A1) 1t follows that $K=1$ whenever $\left|q_{\text {edge }}\right|<1$, so that $\left|z_{n}\right|=1$. The Lyapunov exponent of both matrices is determined by the average of the stochastic variable $r$, and gives, as shown in reference [14], equation (6).

\section{References}

[1] Anderson P. W., Phy's. Rev. 109 (1958) 1492.

[2] Ioffe A F. and Regel A. R., Prog. Semi-conductors 4 (1960) 237.

[3] Abrahams E., anderson P W., Licciardello D. C. and Ramakrishnan T. V., Phw, Rev Lett. 42 (1979) 673.

[4] For a review see Souillard B., Chance and Matter, Les Houches 1986, session XLVI, J Souletie, J. Vannumenus, R. Stora Eds. (North-Holland Physics Publishing, 1986).

[5] Vollhardt D. and Wolfe P., Phys Rev. B 22 (1980) 4666.

[6] Van der Mark M. B., van Albada M. P. and LagendiJk A, Phys Rev. B 37 (1988) 3575

[7] Furstenberg H., Trans. Am. Math. Soc. 108 (1963) 377.

[8] Delyon F., Kunz H. and Souillard B, J. Phys. A 16 (1983) 25

[9] For a review see CARMONA R. and LACROIX J, Spectral Theory of Random Schrodinger Operators (B1rkhäuser Boston, 1983).

[10] Frólich J. and Spencer T., Comm. Math Phys. 88 (1983) 51

[11] KIRSCH W, Schrödinger Operators, H. Holden and A. Jensen Eds. (Springer Verlag. 1988) pp. 264-370.

[12] Simon B., Comm. Math. Phys. 89 (1983) 227.

[13] Thouless D. J., J. Phys. C 5 (1972) 77.

[14] Derrida B and Gardner E., $J$ Phys France 45 (1984) 1283

[15] GENACK A., Scattering and Localization of Classical Waves in Random Media, P Sheng Ed (Singapore. World Scientific, 1990) p. 207.

[16] John S., Comm. Cond. Mat. Phys. 14 (1988) 193

[17] Avishai Y. and Berend D., Phys. Rev. B 41 (1990) 5492.

[18] Kohmoto M., Kadanoff L. P. and Tang C., Phis Rev. Lett 50 (1983) 1870

[19] Megademini T., Phy's. Rev. B 41 (1990) 4693

[20] Gu E., Marr G. V. and Player M. A., Opt. Commun. 77 (1990) 99

[21] Kohmoto M., Sutherland B. and Iguchi K., Phis. Rev Lett 58 (1987) 2436

[22] Kappus M. and Wegner F., Z. Phy's $B 45$ (1981) 15

[23] Lambert C J., Ph.s. Rev $B 29$ (1984) 1091

[24] Saldana X I, Gonzalez de la Cruz G., $J$. Opt Soc. Am 48 (1991) 36

[25] Yoo K. M and Alfano R. R.. Phys Rev B 39 (1989) 5806

[26] Paladin G. and Vulpiani A., Phys. Rev. B 35 (1987) 2015

[27] Parisi G and Vulpiani A, J. Phys. A 19 (1986) L425

[28] Lima R. and Ruffo S., J. Stat. Phy's. 52 (1988) 259.

[29] Bouchaud E and Daoud M., $J$ Phys France 47 (1986) 1467

[30] VERHOEVEN J., private communication (1991)

[31] Nieuwenhulzen Th. M. and Luck J M., J. Stat. Phis. 41 (1985) 745.

[32] CRISANTI A.. J. Phys. A 23 (1990) 5235.

[33] Bruinn M. P., Deposition and Characterisation of Multilayer X-ray Reflection Coatıngs. Ph Dthesis, Vrije Unıversiteit van Amsterdam (1986). 